\title{
The Carrier Transport Properties of B-Doped Si Nanocrystal Films with Various Doping Concentrations
}

\author{
Dan Shan $\mathbb{D}^{1,}, 2$ Yunqing Cao $\mathbb{D},{ }^{2,3}$ Ruihong Yang, ${ }^{1}$ Hongyu Wang, ${ }^{2,4}$ and Tao Tao ${ }^{1}$ \\ ${ }^{1}$ College of Information Engineering, Yangzhou Polytechnic Institute, Yangzhou 225127, China \\ ${ }^{2}$ National Laboratory of Solid State Microstructures and School of Electronic Science and Engineering and Collaborative Innovation \\ Center of Advanced Microstructures, Nanjing University, Nanjing 210093, China \\ ${ }^{3}$ College of Physical Science and Technology, Yangzhou University, Yangzhou 225009, China \\ ${ }^{4}$ Tongda College, Nanjing University of Posts and Telecommunications, Nanjing 210003, China
}

Correspondence should be addressed to Dan Shan; shandnju@126.com and Yunqing Cao; yqcao@yzu.edu.cn

Received 12 June 2020; Accepted 27 July 2020; Published 18 August 2020

Academic Editor: Paul Munroe

Copyright (c) 2020 Dan Shan et al. This is an open access article distributed under the Creative Commons Attribution License, which permits unrestricted use, distribution, and reproduction in any medium, provided the original work is properly cited.

\begin{abstract}
B-doped hydrogenated amorphous silicon $(a-\mathrm{Si}: \mathrm{H})$ films with various doping concentrations were prepared by a plasma-enhanced chemical vapor deposition (PECVD) technique. After thermal annealing, the as-deposited samples, B-doped silicon nanocrystals (Si NCs), were obtained in the films. The electronic properties of B-doped Si NC films with various doping concentrations combined with the microstructural characterization were investigated. A significant improvement of Hall mobility rising to the maximum of $17.8 \mathrm{~cm}^{2} / \mathrm{V} \cdot \mathrm{s}$ was achieved in the Si NC film after B doping, which is due to the reduction of grain boundary (GB) scattering in the B-doped samples. With increasing the doping concentration, it was interesting to find that a metal-insulator transition (MIT) took place in the B-doped Si NC films with high doping concentrations. The different carrier transport properties in the B-doped Si NC films with various doping concentrations were investigated and further discussed with emphasis on the scattering mechanisms in the transport process.
\end{abstract}

\section{Introduction}

Si nanocrystals (Si NCs) have attracted more and more interest in recent years because of their possible applications in many kinds of nanoelectronic and optoelectronic devices such as light emitter devices, thin-film solar cells, photodetectors, and synaptic devices [1-8]. For example, highly efficient electroluminescence devices based on Si NCs have been fabricated by Yao et al. [9]. It was reported that the maximum external quantum efficiency and largest optical power density of the device reached as high as $2.7 \%$ and $0.11 \mathrm{~mW} / \mathrm{cm}^{2}$, respectively. Recently, Si NCs have been utilized to incorporate with organic semiconductors or lead halide perovskites, which can improve the power conversion efficiency of solar cells [10-12]. In order to further improve the performance of devices based on Si NCs, active doping is usually required to obtain all kinds of desired properties of the Si NC materials. However, doping in Si NCs is quite different from their bulk counterpart due to the so-called "self-purification effect," and the states of doped atoms in $\mathrm{Si}$ NCs such as bonding structures, sites, and electrical activities are usually influenced by the microstructures $[13,14]$. With the advances in the synthesis of B- and P-doped Si NCs, several researchers have reported successful doping in $\mathrm{Si}$ $\mathrm{NCs}$, offering significant freedom in tuning the electrical properties of Si NCs [15-18]. But a comparable study on the carrier transport properties in doped Si NCs is still lacking. Therefore, further understanding of the fundamental carrier transport properties in doped Si NCs film is necessary as various theoretical aspects and the mechanisms of dopant incorporation are still under debate [19-22].

In our previous works, undoped, B-doped, and P-doped Si NC films were prepared and their electronic properties were comparably studied. It could be found that very high room temperature conductivities were achieved both in Si NC films after $\mathrm{B}$ and $\mathrm{P}$ doping, which demonstrates that doping is an effective way to improve the electronic properties of Si NCs for device applications [23]. It was also found that the carrier 
mobility was gradually improved in P-doped Si NC films compared with the undoped one due to the reduction of barrier height of grain boundaries (GBs) and the passivation of surface defect states after P doping [24]. In the present work, the carrier transport properties in B-doped Si NC films with various doping concentrations were investigated via the temperature-dependent Hall effect measurements. A significant improvement of Hall mobility was still achieved after $\mathrm{B}$ doping due to the reduction of barrier height of GBs in the films, which can be well described by the previously proposed model. It was interesting to find that a metal-insulator transition (MIT) took place in the B-doped samples during further increasing the doping concentration. The different carrier transport properties together with scattering mechanisms in the transport process in the B-doped samples with various doping concentrations were investigated and discussed.

\section{Experiment}

The B-doped hydrogenated amorphous silicon $(a-\mathrm{Si}: \mathrm{H})$ films were prepared by a plasma-enhanced chemical vapor deposition (PECVD) method using a gas mixture of pure silane $\left(\mathrm{SiH}_{4}\right)$, hydrogen $\left(\mathrm{H}_{2}\right)$, and diborane $\left(\mathrm{B}_{2} \mathrm{H}_{6}, 1 \%\right.$ diluted in $\left.\mathrm{H}_{2}\right)$. The flow rate of $\mathrm{SiH}_{4}\left(F_{\mathrm{S}}\right)$ was kept at $5 \mathrm{sccm}$ (standard cubic centimeter per minute). Boron concentrations were then changed by adjusting the flow rate of $\mathrm{B}_{2} \mathrm{H}_{6}\left(F_{\mathrm{B}}\right)$, which is controlled at $0 \mathrm{sccm}$ (undoped), $0.5 \mathrm{sccm}, 1 \mathrm{sccm}$, and $5 \mathrm{sccm}$, respectively. The gas-chamber pressure, substrate temperature, and radio frequency power were $10 \mathrm{mTorr}$, $250^{\circ} \mathrm{C}$, and $30 \mathrm{~W}$ during the growth process, respectively. After deposition, the samples, all which show the thickness of about $180 \mathrm{~nm}$, were subsequently annealed in a conventional furnace at temperatures of $1000^{\circ} \mathrm{C}$ for $1 \mathrm{~h}$ in nitrogen ambient. Quartz plates and monocrystalline $\mathrm{Si}$ wafers were used as substrates for the various measurements.

Raman scattering spectra were detected by a Jobin Yvon Horiba HR800 spectrometer operating with $1800 \mathrm{~g} / \mathrm{mm}$ grating, where the excitation light source is $\mathrm{Ar}+$ laser with a wavelength of $514 \mathrm{~nm}$. High-resolution transmission electron microscopy (TEM) images were observed by a TECNAI G2F20 FEI high-resolution transmission electron microscopy. The bonding configurations were characterized by PHI 5000 Versa Probe X-ray photoelectron spectroscopy (XPS), and the composition signal was detected after Ar+ etching to $5 \mathrm{~nm}$. Temperature-dependent Hall measurements were measured by the LakeShore 8400 Hall effect measurement system using van der Pauw (VDP) geometry. All the samples were heated at $400 \mathrm{~K}$ for $30 \mathrm{~min}$ and then measured back to $300 \mathrm{~K}$ with the step of $10 \mathrm{~K}$ in argon ambient.

\section{Results and Discussion}

Raman spectroscopy was utilized to investigate the change of microstructures in the B-doped Si NC films with various doping concentrations. The signals at 480 and $520 \mathrm{~cm}^{-1}$ represent the transverse-optical (TO) vibration mode of amorphous silicon and crystalline silicon, respectively. As shown in Figure 1, all the samples show a sharp and strong peak close to $520 \mathrm{~cm}^{-1}$, which indicates a pure Si crystalline

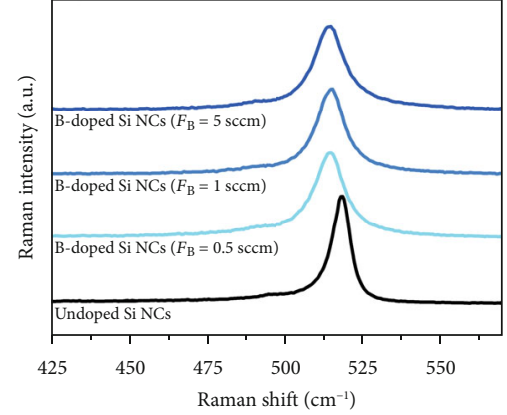

FIgURE 1: Raman spectroscopy of B-doped Si NC films with various doping concentrations.

structure. The crystalline fraction, $X_{c}$, can be determined based on Raman spectra according to the formula $X_{c}=I_{c} /$ $\left(I_{\mathrm{c}}+0.88 I_{\mathrm{a}}\right)$, where $I_{\mathrm{c}}$ and $I_{\mathrm{a}}$ are the crystalline and amorphous parts of the integrated Raman scattering intensity, respectively [25]. It is found that $X_{c}$, which is about $85 \%$ for the undoped sample, decreases to $80 \%$ after B doping. Similar results were also reported by our previous work [26]. It can be implied that the reduction of $X_{c}$ is attributed to the introduction of $B$ dopants, which leads to an increase of bond-angle and bond-length fluctuations as the B dopant incorporation in the films and causes the degradation of the short-range order [27]. Furthermore, the size of Si NCs (R) before and after $\mathrm{B}$ doping can be roughly estimated from the shift between $\omega_{1}$ and $\omega_{\text {LO-TO }}$ using the bending parameters of bulk optical phonon dispersion curve $\beta_{\mathrm{LO}-\mathrm{TO}}$, according to the formula $\omega_{1}^{2}=\omega_{\mathrm{LO}-\mathrm{TO}}^{2}-\beta_{\mathrm{LO}-\mathrm{TO}}(\pi / R)^{2}$, where $\omega_{1}$ and $\omega_{\text {LO-TO }}$ are the Raman spectra of Si NC films and bulk Si, respectively [28]. We worked out that the average size of Si NCs is about $24 \mathrm{~nm}$ for the undoped sample and $13 \mathrm{~nm}$ for the samples after B doping. From the above discussion, it can be confirmed that the introduction of B dopants will degrade the Si NCs and generates a reduction of crystallization and grain size in the films.

The microstructures of Si NC films before and after B doping were further investigated using the cross-sectional TEM observations. The sample thickness is clearly observed about $180 \mathrm{~nm}$ as shown in Figure 2(a), which is in good agreement with the predesigned value estimated by the deposition rate. The formation of Si NCs can be further identified in the high-resolution TEM image as given in Figure 2(b). A large number of Si NCs with the grain size of more than $20 \mathrm{~nm}$ spread over the undoped Si NC film, indicating a good crystallization of the film. However, the grain size of $\mathrm{Si}$ NCs is decreased to only about $10 \mathrm{~nm}$ after B doping as shown in Figure 2(c), which is in accordance with the estimate from Raman results. Compared with the undoped Si NC film, it can be clearly seen that the B-doped sample has a relatively low density due to the degradation of the film after B doping.

The chemical bonding environments of B-doped Si NC films were then investigated by XPS. The B 1s peaks of all the B-doped Si NC films are shown in Figure 3. For the Bdoped samples, the peak around $186 \mathrm{eV}$ can be attributed to the $\mathrm{B}-\mathrm{Si}$ signal [29]. It can be clearly identified that the 


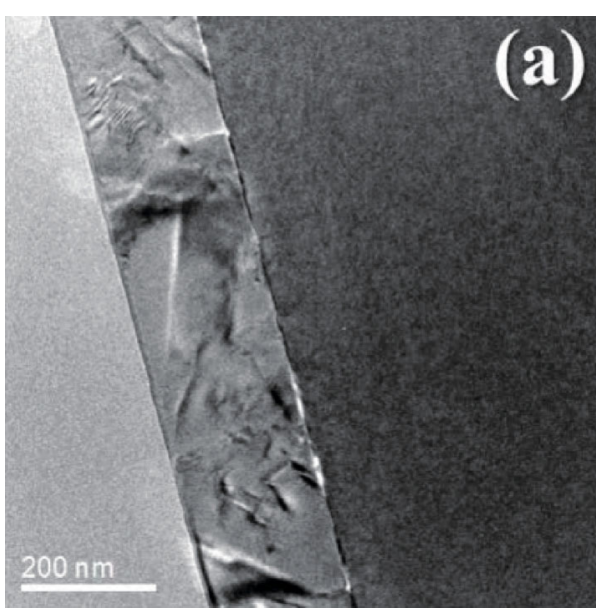

(a)

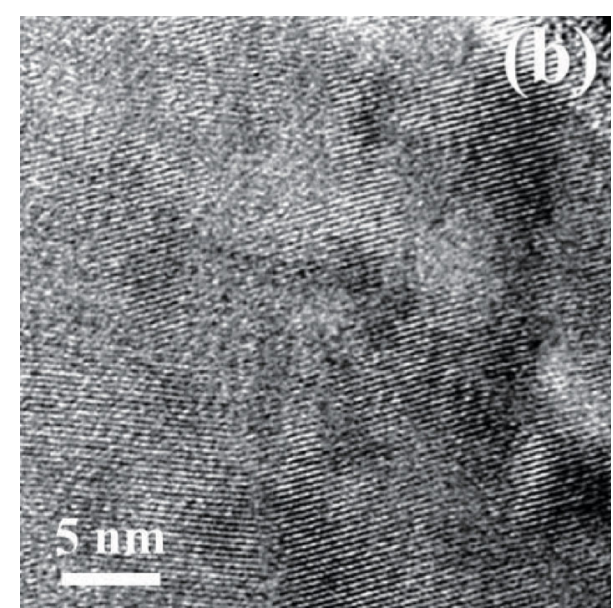

(b)

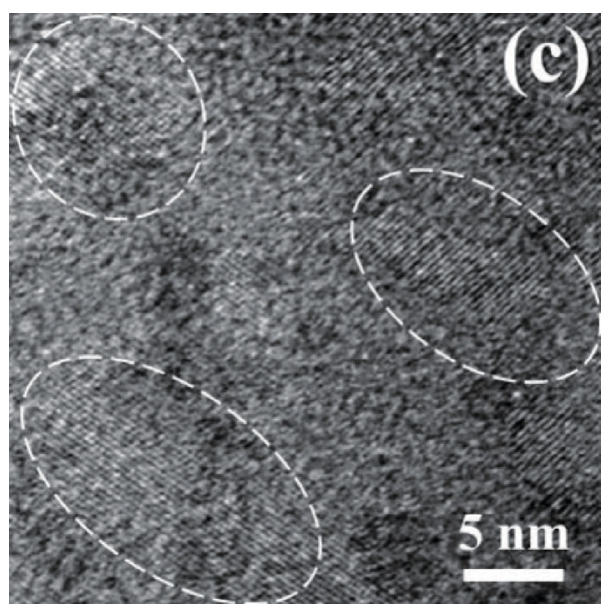

(c)

Figure 2: Cross-sectional TEM images of Si NC films before and after B doping.

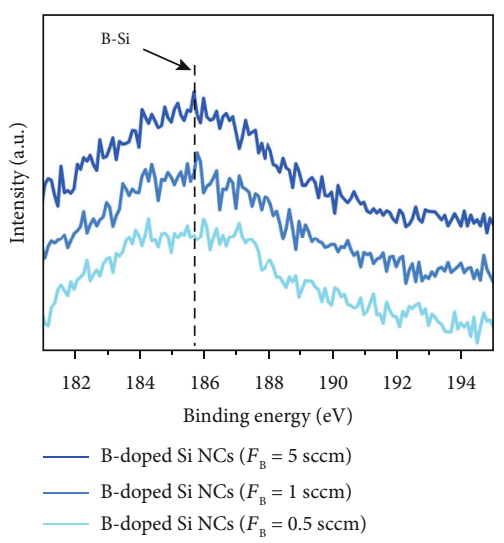

FIgUre 3: The XPS of B-doped Si NC films with $F_{\mathrm{B}}=0.5,1$, and $5 \mathrm{sccm}$.

intensity of the B-Si peak is not so weak and increases slightly with $\mathrm{B}$ doping concentrations. The $\mathrm{B}$ atom contents are estimated to be $0.31 \%, 0.51 \%$, and $0.57 \%$ for the B-doped samples with $F_{\mathrm{B}}=0.5 \mathrm{sccm}, 1 \mathrm{sccm}$, and $5 \mathrm{sccm}$, respectively.
TABLE 1: Room temperature Hall measurement of B-doped Si NC films with various doping concentrations.

\begin{tabular}{|c|c|c|}
\hline Sample & Carrier concentration & Dark conductivity \\
\hline Undoped Si NCs & $3.4 \times 10^{12} \mathrm{~cm}^{-3}$ & $8.6 \times 10^{-7} \mathrm{~S} \cdot \mathrm{cm}^{-1}$ \\
\hline $\begin{array}{l}\text { B-doped Si NCs } \\
\left(F_{\mathrm{B}}=0.5 \mathrm{sccm}\right)\end{array}$ & $1 \times 10^{20} \mathrm{~cm}^{-3}$ & $174 \mathrm{~S} \cdot \mathrm{cm}^{-1}$ \\
\hline $\begin{array}{l}\text { B-doped Si NCs } \\
\left(F_{\mathrm{B}}=1 \mathrm{sccm}\right)\end{array}$ & $1.2 \times 10^{20} \mathrm{~cm}^{-3}$ & $272 \mathrm{~S} \cdot \mathrm{cm}^{-1}$ \\
\hline $\begin{array}{l}\text { B-doped Si NCs } \\
\left(F_{\mathrm{B}}=5 \mathrm{sccm}\right)\end{array}$ & $1.6 \times 10^{20} \mathrm{~cm}^{-3}$ & $456 \mathrm{~S} \cdot \mathrm{cm}^{-1}$ \\
\hline
\end{tabular}

It was reported that the $\mathrm{B}$ doping ratio is about $0.5 \%$ in $\mathrm{Si}$ NCs with $F_{\mathrm{S}}: F_{\mathrm{B}}=2: 1 \quad\left(F_{\mathrm{S}}=31.5 \mathrm{sccm}\right.$ and $F_{\mathrm{B}}=15.2$ $\mathrm{sccm}$ ) [15], which is in the same order as in our case.

The electrical properties of B-doped Si NC films with various doping concentrations were investigated by Hall effect measurements. Room temperature dark conductivities and carrier concentrations are exhibited in Table 1. The carrier concentration, which is about $1.7 \times 10^{12} \mathrm{~cm}^{-3}$ for the undoped sample, is significantly increased to $10^{20} \mathrm{~cm}^{-3}$ order after B doping, indicating that the Si NC samples are easily 


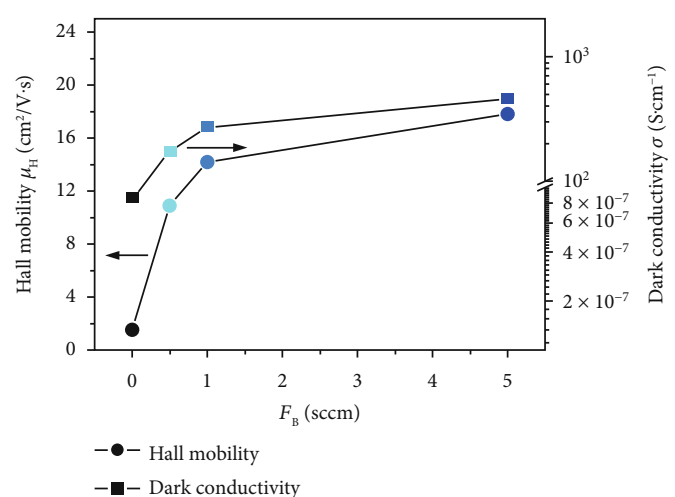

FIgURE 4: The carrier mobilities and dark conductivities of B-doped Si NC films with $F_{\mathrm{B}}=0.5,1$, and $5 \mathrm{sccm}$.

heavily doped even at a relatively low gas doping ratio. It can be understood that the doping concentration can reach a very high value even if only a few dopants are incorporated into a one-Si dot with a diameter on the nanometer scale. Meanwhile, the dark conductivity, which is strongly determined by the carrier concentration, is increased from $8.6 \times 10^{-7}$ $\mathrm{S} \cdot \mathrm{cm}^{-1}$ for the undoped sample to the maximum of $456 \mathrm{~S} \cdot \mathrm{cm}^{-1}$ for the B-doped sample with the highest doping concentration $\left(F_{\mathrm{B}}=5 \mathrm{sccm}\right)$.

The carrier mobilities of B-doped Si NC films with various doping concentrations are shown in Figure 4. It is very interesting to find that the Hall mobility is obviously improved after B doping. The Hall mobility, which is only $1.6 \mathrm{~cm}^{2} / \mathrm{V} \cdot \mathrm{s}$ for the undoped sample, is enhanced to $10.9 \mathrm{~cm}^{2} / \mathrm{V} \cdot \mathrm{s}$ for the B-doped sample with $F_{\mathrm{B}}=0.5 \mathrm{sccm}$. As we know, the carrier mobility usually reduces after introducing the dopants in bulk Si due to the strong scattering effect of impurities. Here, the abnormal behavior of carrier mobility must be caused by other reasons. In our previous works, the similar behavior of carrier mobility was also found in the P-doped Si NC films [24]. In fact, the carrier mobility is usually low in Si NC films due to the strong scattering from the GBs. It was reported that a large amount of GBs exist in the Si NC films, which contain a higher concentration of defects than the grains themselves, leading to carrier trapping at GBs [30]. After trapping the carriers, the traps become electrically charged, producing a potential energy barrier which impedes the carriers moving from one crystallite to another [31, 32]. However, the barrier height at GBs can be reduced obviously if the carrier concentration is high enough, which contributes to a decrease of scattering from the GBs [22]. Thus, the significant increase of mobility after B doping can be explained by the reduced barrier height of GBs in the B-doped samples with a high carrier concentration (the carrier concentration reaches $10^{20} \mathrm{~cm}^{-3}$ order). Moreover, further increasing the $\mathrm{B}$ doping concentration can still increase the Hall mobility, which reaches about $17.8 \mathrm{~cm}^{2} / \mathrm{V} \cdot \mathrm{s}$ in the B-doped sample with $F_{\mathrm{B}}=5 \mathrm{sccm}$. According to Seto's theory, the potential barrier height of GBs in the high-doped Si NC films can be estimated by the equation $V_{\mathrm{B}}=q Q_{\mathrm{t}}^{2} / 8 \varepsilon N$, where $V_{\mathrm{B}}$ is the potential barrier height at GBs, $Q_{\mathrm{t}}$ is the GBs trapping state density, and $N$ is the carrier concentration,

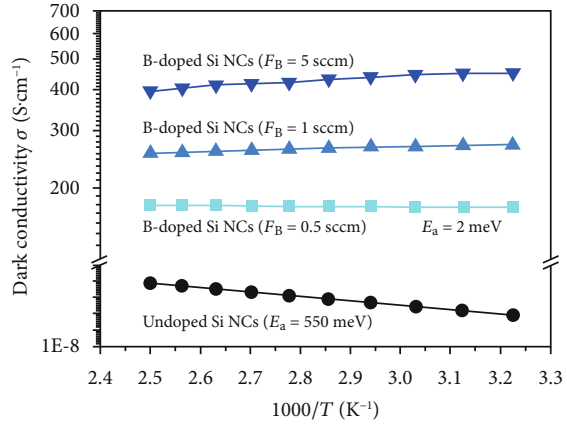

Figure 5: Temperature-dependent dark conductivities of B-doped Si NC films with various doping concentrations.

respectively [30]. It can be deduced that the potential barrier height $V_{\mathrm{B}}$ is decreased with increasing the carrier concentration $N$. Consequently, the mobility of the B-doped Si NC film can be increased by further increasing the doping concentration, which finally is more than ten times higher than that of the undoped one.

In order to give a deeper understanding of the carrier transport properties in B-doped Si NC films, temperaturedependent dark conductivities were measured for all the samples within the temperature range from $300 \mathrm{~K}$ to $400 \mathrm{~K}$. As shown in Figure 5, all the samples can be well fitted by the Arrhenius plots:

$$
\sigma=\sigma_{0} \exp \left(-\frac{E_{\mathrm{a}}}{k_{\mathrm{B}} T}\right),
$$

where $\sigma_{0}$ is the conductivity prefactor, $k_{\mathrm{B}}$ is Boltzmann's constant, and $E_{\mathrm{a}}$ is the conductivity activation energy. The conductivity activation energy $E_{\mathrm{a}}$ can be obtained using the slope of $\ln \sigma$ versus the $1 / T$ curve. For the undoped sample, the value of $E_{\mathrm{a}}$ is $0.55 \mathrm{eV}$ which implies the Fermi level locating at the midgap of the Si NCs. After B doping, $E_{\mathrm{a}}$ is rapidly decreased to $2 \mathrm{meV}$ for the low-doped sample $\left(F_{\mathrm{B}}=0.5\right.$ sccm), which suggests that the Fermi level shifts to near the top of the valence band due to the doping effect. It can be found that $\sigma$ versus $1000 / T$ exhibits a linear relation in the whole measurement temperature range for the un- and low-doped samples, which means the thermally activated transport dominating the carrier transport processes [33]. However, for the high-doped Si NC films $\left(F_{\mathrm{B}}=1 \mathrm{sccm}\right.$ and $5 \mathrm{sccm})$, it is interesting to find that the dark conductivity is decreased with temperature increasing, which exhibits metal-like properties. Chen et al. reported that the metalinsulator transition (MIT) usually occurs in semiconductor NC films at some critical carrier concentration. The MIT criterion can be described by the following equation:

$$
n_{\mathrm{c}} \rho^{3} \simeq 0.3 \mathrm{~g}
$$

where $n_{c}$ is the critical carrier concentration, $g$ is the number of equivalent minima in the conduction band of the semiconductor, and $\rho$ is the touching facets of the radius between NCs. Here, the facet radius $\rho$ can be approximated as $\rho=$ 


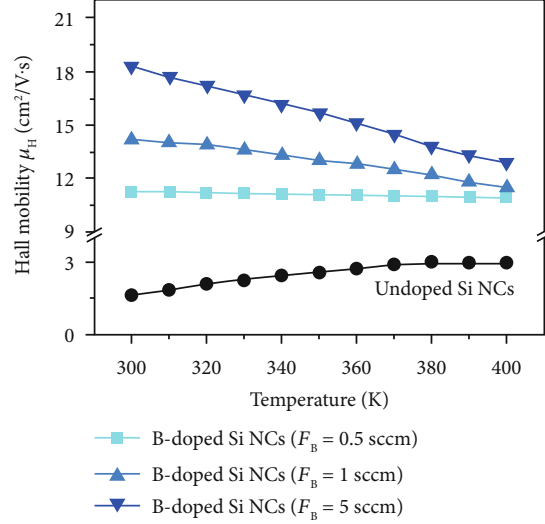

(a)

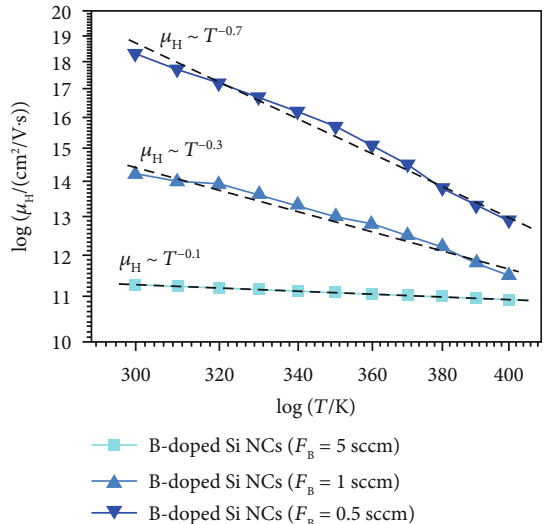

(b)

FIgure 6: (a) Temperature-dependent Hall mobilities of B-doped Si NC films with various doping concentrations and (b) the Hall mobility, $\mu_{\mathrm{H}}$, as a function of temperature for the B-doped samples. The lines represent least-squares fits to $\mu_{\mathrm{H}}(T) \propto T^{n}$.

$\sqrt{d a / 2}$, where $a$ is the lattice constant and $d$ is the NC diameter [17]. In the present work, the NC diameter $d$ for the Bdoped sample is about $13 \mathrm{~nm}$ from the Raman results. For such value of $d$, we predicted $n_{\mathrm{c}} \simeq 1.17 \times 1020 \mathrm{~cm}^{-3}$, which just exceeds the carrier concentration of B-doped samples with $F_{\mathrm{B}}=0.5 \mathrm{sccm}$ as shown in Table 1 . It can be concluded that the metal-insulator transition took place in the B-doped Si NC films with $F_{\mathrm{B}}=1 \mathrm{sccm}$ and $5 \mathrm{sccm}$, leading to a different behavior of $\sigma-T$ relationship.

For further surveying the scattering mechanisms in the carrier transport process, the temperature-dependent Hall mobilities of $\mathrm{B}$-doped $\mathrm{Si} \mathrm{NC}$ films with various doping concentrations were investigated as shown in Figure 6(a). For the undoped sample, the increase of carrier mobility with temperature indicates a strong scattering from the GBs in $\mathrm{Si}$ NC film, which was expounded in detail in our previous work [34]. However, the GB scattering will be reduced after B doping according to the above discussion, and the temperaturedependent behavior of mobilities exhibits a different trend in B-doped samples, especially in the high-doped samples $\left(F_{\mathrm{B}}=1 \mathrm{sccm}\right.$ and $\left.5 \mathrm{sccm}\right)$. It can be found that the mobilities decrease with increasing temperature. Combining the reduction of conductivities with increasing temperature, we can confirm that the band-like transport behaviors take place in the high-doped Si NC films [35, 36]. Furthermore, the scattering mechanisms can be investigated by the equation $\mu_{\mathrm{H}}(T) \propto T^{n}$, where $n$ can be estimated by $\log \mu_{\mathrm{H}}$ plotted as a function of $\log T$. Least-squares fits of the data yield $n$ $=-0.1,-0.3$, and -0.7 for the B-doped Si NC films with $F_{\mathrm{B}}=0.5 \mathrm{sccm}, 1 \mathrm{sccm}$, and $5 \mathrm{sccm}$ given in Figure $6(\mathrm{~b})$, respectively. In $\mathrm{c}-\mathrm{Si}$, typical scattering mechanisms are the scattering at acoustic phonons and neutral impurities that yield values of -1.5 and $\approx 0$. It can be suggested that a superposition of neutral impurity scattering, acoustic phonon scattering, and weak GB scattering dominates the carrier transport process in B-doped $\mathrm{Si} \mathrm{NC}$ films. With the increase of $\mathrm{B}$ doping concentration, the $n$-value is gradually decreased to near -1.5 , which implies that the acoustic phonon scattering increasingly plays an important role in the carrier transport process.

\section{Conclusion}

In summary, B-doped $\mathrm{Si} \mathrm{NC}$ films with various doping concentrations were prepared and their carrier transport properties together with the microstructures were investigated. The electrical properties of Si NC films after B doping are significantly improved compared with the undoped one. Room temperature Hall mobility and conductivity are increased with increasing the doping concentration and reach $17.8 \mathrm{~cm}^{2} / \mathrm{V} \cdot \mathrm{s}$ and $456 \mathrm{~S} \cdot \mathrm{cm}^{-1}$ for the B-doped Si NC film with $F_{\mathrm{B}}=5 \mathrm{sccm}$. The enhanced Hall mobility after B doping can be ascribed to the reduced scattering from the GBs. Based on the temperature-dependent Hall effect measurement, it can be found that a MIT occur in the B-doped Si NC films with $F_{\mathrm{B}}=1 \mathrm{sccm}$ and $5 \mathrm{sccm}$. As a consequence, the carrier transport mechanism is correspondingly changed from the thermally activated transport in the B-doped samples at low doping concentration to the band-like carrier transport in the B-doped samples at high doping concentration. Meanwhile, the acoustic phonon scattering gradually dominates the carrier transport process in the B-doped Si NC films with the increase of doping concentration.

\section{Abbreviations}

a-Si:H: Hydrogenated amorphous silicon

PECVD: Plasma-enhanced chemical vapor deposition

Si NCs: Silicon nanocrystals

GBs: Grain boundaries

MIT: Metal-insulator transition

TEM: Transmission electron microscopy

XPS: X-ray photoelectron spectroscopy.

\section{Data Availability}

The data used to support the findings of this study are available from the corresponding authors upon request.

\section{Conflicts of Interest}

The authors declare that they have no conflicts of interest. 


\section{Authors' Contributions}

Dan Shan and Yunqing Cao conceived the idea and carried out the experiments. Dan Shan, Hongyu Wang, and Tao Tao participated in the preparation of the samples. Dan Shan, Ruihong Yang, and Yunqing Cao took part in the experiments and the discussion of the results. Dan Shan and Yunqing Cao drafted the manuscript with the instruction of Hongyu Wang, Tao Tao, and Ruihong Yang. All authors read and approved the final manuscript.

\section{Acknowledgments}

This work was supported by the NSFC (grant numbers 61704148 and 51979077), China Postdoctoral Science Foundation (grant number 2019M651750), Overseas Study Program for Outstanding Young and Middle-aged Teachers and Principals of Universities in Jiangsu Province, Joint Project of Industry-University-Research of Jiangsu Province (grant number BY2019248), "Qing Lan Project" of Jiangsu Province (2018 and 2020), NSF of Jiangsu Province (grant number BK20170514), Natural Science Foundation of the Jiangsu Higher Education Institutions of China (grant numbers 17KJB140030 and 18KJB510035), Fundamental Research Funds for the Central Universities (grant number 2019B44214), PAPD, Research Fund of Nanjing University of Posts and Telecommunications (grant number NY218094), Open Project of State Key Laboratory of Solid State Microstructure Physics (grant numbers M31037 and M31038), and Key R\&D Plan of Yangzhou City (grant number YZ2018072).

\section{References}

[1] S. Zhao, X. Liu, X. Pi, and D. Yang, "Light-emitting diodes based on colloidal silicon quantum dots," Journal of Semiconductors, vol. 39, no. 6, article 061008, 2018.

[2] J. López-Vidrier, S. Gutsch, O. Blázquez et al., "Effect of $\mathrm{Si}_{3} \mathrm{~N}_{4}$ mediated inversion layer on the electroluminescence properties of silicon nanocrystal superlattices," Advanced Electronic Materials, vol. 4, no. 5, article 1700666, 2018.

[3] X. Liu, Y. Ji, Z. Lu et al., "Enhanced device performance of Si nanowires/Si nanocrystals heterojunction solar cells with ultrathin $\mathrm{Al}_{2} \mathrm{O}_{3}$ passivation," Physica E: Low-dimensional Systems and Nanostructures, vol. 120, article 114048, 2020.

[4] C. Wen, Y. J. Yang, Y. J. Ma et al., "Sulfur-hyperdoped silicon nanocrystalline layer prepared on polycrystalline silicon solar cell substrate by thin film deposition and nanosecond-pulsed laser irradiation," Applied Surface Science, vol. 476, pp. 4960, 2019.

[5] T. Yu, F. Wang, Y. Xu, L. L. Ma, X. D. Pi, and D. Yang, "Graphene coupled with silicon quantum dots for highperformance bulk-silicon-based Schottky-junction photodetectors," Advanced Materials, vol. 28, no. 24, pp. 49124919, 2016.

[6] R. A. John, F. Liu, N. A. Chien et al., "Synergistic gating of electro-iono-photoactive 2D chalcogenide neuristors: coexistence of Hebbian and homeostatic synaptic metaplasticity," Advanced Materials, vol. 30, no. 25, article 1800220, 2018.

[7] Y. Xu, A. Ali, K. Shehzad et al., "Solvent-based soft-patterning of graphene lateral heterostructures for broadband high-speed metal-semiconductor-metal photodetectors," Advanced Materials Technologies, vol. 2, no. 2, article 1600241, 2017.

[8] A. Ali, K. Shehzad, H. W. Guo et al., "High-performance, flexible graphene/ultra-thin silicon ultra-violet image sensor," in IEEE International Electron Devices Meeting, pp. 8.6.1-8.6.4, San Francisco, CA, USA, 2017.

[9] L. Yao, T. Yu, L. Ba et al., "Efficient silicon quantum dots light emitting diodes with an inverted device structure," Journal of Materials Chemistry C, vol. 4, no. 4, pp. 673-677, 2016.

[10] L. Lu, M. A. Kelly, W. You, and L. Yu, "Status and prospects for ternary organic photovoltaics," Nature Photonics, vol. 9, no. 8, pp. 491-500, 2015.

[11] S. Zhao, X. Pi, C. Mercier, Z. Yuan, B. Sun, and D. Yang, "Silicon-nanocrystal-incorporated ternary hybrid solar cells," Nano Energy, vol. 26, pp. 305-312, 2016.

[12] C. Rocks, V. Svrcek, T. Velusamy, M. Macias-Montero, P. Maguire, and D. Mariotti, "Type-I alignment in $\mathrm{MAPbI}_{3}$ based solar devices with doped-silicon nanocrystals," Nano Energy, vol. 50, pp. 245-255, 2018.

[13] G. M. Dalpian and J. R. Chelikowsky, "Self-purification in semiconductor nanocrystals," Physical Review Letters, vol. 96, no. 22, article 226802, 2006.

[14] Z. Ni, S. Zhou, S. Zhao, W. Peng, D. Yang, and X. Pi, "Silicon nanocrystals: unfading silicon materials for optoelectronics," Materials Science and Engineering: R: Reports, vol. 138, pp. 85-117, 2019.

[15] S. Zhou, X. Pi, Z. Ni et al., "Boron and phosphorushyperdoped silicon nanocrystals," Particle \& Particle Systems Characterization, vol. 32, no. 2, pp. 213-221, 2015.

[16] E. Thimsen, "Beyond equilibrium thermodynamics in the low temperature plasma processor," Journal of Vacuum Science \& Technology B, vol. 36, no. 4, article 048501, 2018.

[17] T. Chen, K. V. Reich, N. J. Kramer, H. Fu, U. R. Kortshagen, and B. I. Shklovskii, "Metal-insulator transition in films of doped semiconductor nanocrystals," Nature Materials, vol. 15, no. 3, pp. 299-303, 2016.

[18] S. Gutsch, J. Laube, D. Hiller et al., "Electronic properties of phosphorus doped silicon nanocrystals embedded in $\mathrm{SiO}_{2}$," Applied Physics Letters, vol. 106, no. 11, article 113103, 2015.

[19] M. Xie, D. Li, L. Chen, F. Wang, X. Zhu, and D. Yang, "The location and doping effect of boron in Si nanocrystals embedded silicon oxide film," Applied Physics Letters, vol. 102, no. 12, article 123108, 2013.

[20] M. Perego, C. Bonafos, and M. Fanciulli, "Phosphorus doping of ultra-small silicon nanocrystals," Nanotechnology, vol. 21, no. 2, article 025602, 2010.

[21] M. Fujii, H. Sugimoto, M. Hasegawa, and K. Imakita, "Silicon nanocrystals with high boron and phosphorus concentration hydrophilic shell-Raman scattering and X-ray photoelectron spectroscopic studies," Journal of Applied Physics, vol. 115, no. 8, article 084301, 2014.

[22] B. P. Veettil, L. Wu, X. Jia et al., "Passivation effects in B doped self-assembled Si nanocrystals," Applied Physics Letters, vol. 105, no. 22, article 222108, 2014.

[23] D. Shan, M. Q. Qian, Y. Ji, X. F. Jiang, J. Xu, and K. J. Chen, "The change of electronic transport behaviors by $\mathrm{P}$ and $\mathrm{B}$ doping in nano-crystalline silicon films with very high conductivities," Nanomaterials, vol. 6, no. 12, p. 233, 2016.

[24] D. Shan, Y. Ji, D. K. Li et al., "Enhanced carrier mobility in Si nano-crystals via nanoscale phosphorus doping," Applied Surface Science, vol. 425, pp. 492-496, 2017. 
[25] R. Tsu, J. Gonzalez-Hernandez, S. S. Chao, S. C. Lee, and K. Tanaka, "Critical volume fraction of crystallinity for conductivity percolation in phosphorus-doped Si:F:H alloys," Applied Physics Letters, vol. 40, no. 6, pp. 534-535, 1982.

[26] C. Song, X. Wang, R. Huang, J. Song, and Y. Q. Guo, "Effects of doping concentration on the microstructural and optoelectrical properties of boron doped amorphous and nanocrystalline silicon films," Materials Chemistry and Physics, vol. 142, no. 1, pp. 292-296, 2013.

[27] R. Saleh and N. H. Nickel, "The influence of boron concentrations on structural properties in disorder silicon films," Applied Surface Science, vol. 254, no. 2, pp. 580-585, 2007.

[28] P. Caldelas, A. G. Rolo, M. J. M. Gomes et al., "Raman and XRD studies of Ge nanocrystals in alumina films grown by RF-magnetron sputtering," Vacuum, vol. 82, no. 12, pp. 1466-1469, 2008.

[29] R. J. Ward and B. J. Wood, "A comparison of experimental and theoretically derived sensitivity factors for XPS," Surface and Interface Analysis, vol. 18, no. 9, pp. 679-684, 1992.

[30] J. Y. W. Seto, "The electrical properties of polycrystalline silicon films," Journal of Applied Physics, vol. 46, no. 12, pp. 5247-5254, 1975.

[31] C. H. Seager and T. G. Castner, "Zero-bias resistance of grain boundaries in neutron-transmutation-doped polycrystalline silicon," Journal of Applied Physics, vol. 49, no. 7, pp. 38793889, 1978.

[32] W. Hellmich, G. Muller, G. Krotz, G. Derst, and S. Kalbitzer, "Optical absorption and electronic transport in ionimplantation-doped polycrystalline SiC films," Applied Physics A, vol. 61, no. 2, pp. 193-201, 1995.

[33] S. Y. Myong, K. S. Lim, and M. Konagai, "Effect of hydrogen dilution on carrier transport in hydrogenated boron-doped nanocrystalline silicon-silicon carbide alloys," Applied Physics Letters, vol. 88, no. 10, article 103120, 2006.

[34] D. Shan, Y. Ji, J. Xu et al., "Microstructure and carriertransport behaviors of nanocrystalline silicon thin films annealed at various temperatures," Physica Status Solidi (a), vol. 213, no. 7, pp. 1675-1679, 2016.

[35] J. S. Lee, M. V. Kovalenko, J. Huang, D. S. Chung, and D. V. Talapin, "Band-like transport, high electron mobility and high photoconductivity in all-inorganic nanocrystal arrays," Nature Nanotechnology, vol. 6, no. 6, pp. 348-352, 2011.

[36] E. Talgorn, Y. Gao, M. Aerts et al., "Unity quantum yield of photogenerated charges and band-like transport in quantumdot solids," Nature nanotechnology, vol. 6, no. 11, pp. 733739,2011 